\title{
Pancreatic endocrine tumour with cytoplasmic keratin whorls. Is the term "rhabdoid" appropriate?
}

\author{
R Chetty, S L Asa
}

J Clin Pathol 2004;57:1106-1110. doi: 10.1136/icp.2004.018309

A 50 year old woman presented with acute abdominal pain accompanied by nausea and vomiting and was found to have a mass in the head of the pancreas by imaging. The clinical impression was of a pancreatic carcinoma and a Whipple's procedure was performed. Microscopic examination of the tumour showed it to be a low grade neuroendocrine carcinoma arranged in a tubuloacinar or tubulopapillary pattern, and composed of cells harbouring very prominent intracytoplasmic inclusions. These inclusions varied in appearance from being pale pink and hyaline in quality to more eosinophilic and globular causing displacement of the nucleus. Ultrastructural examination showed typical paranuclear aggregates of intermediate filaments. Inclusions of this type have been described previously as "signet ring like" and "rhabdoid". It was felt that the inclusions more closely resemble the fibrous bodies that are seen in pituitary adenomas. In addition, it is suggested that both signet ring and rhabdoid are not appropriate because they do not reflect histogenesis and are not necessarily reflective of tumour biology. It is suggested that the term "cytokeratin aggresomes" should be used to describe this distinctive phenotype.

$\mathrm{P}$ ancreatic endocrine tumours (PETs) are usually composed of uniform cells with eosinophilic, granular cytoplasm and nuclei containing chromatin with a "salt and pepper" distribution. However, endocrine cells encountered in these lesions may show variable morphological alterations, including oncocytic change, ${ }^{12}$ clear cytoplasm, ${ }^{34}$ rhabdoid features, ${ }^{56}$ small intracytoplasmic eosinophilic globules, ${ }^{7}$ mucin accumulation resulting in goblet cell formation, ${ }^{89}$ and spindle cell morphology. ${ }^{70}$ An extremely rare variant is the so called "signet ring" cell variant, which was described by Stokes et al in a non-functioning PET. ${ }^{71}$ The collection of variants of this lesion expands with each case report, but the importance of each remains unknown.

"The term rhabdoid, meaning rhabdomyoblast like, implies a resemblance to rhabdomyoblasts that is characteristic of aggressive, dedifferentiated malignancies" $^{\prime \prime}$

We describe a pancreatic endocrine tumour composed of cells containing distinct intracytoplasmic hyaline appearing inclusion bodies. There was a superficial resemblance to signet ring cells because several of the inclusions were pale and clear. However, we feel that this term is misleading and is a misnomer, because the use of the term "signet ring" cell implies the presence of intracytoplasmic mucin vacuoles. The tumour cells had a homogeneous pale space within the cytoplasm on haematoxylin and eosin (H\&E) staining, similar to those identified in so called "rhabdoid" tumours. ${ }^{56}$ This term also is not ideal, and is confusing because the term "rhabdoid", meaning rhabdomyoblast like, implies a resemblance to rhabdomyoblasts that is characteristic of aggressive, dedifferentiated malignancies.

The cytoplasmic hyaline globules encountered in our patient and the other so called "rhabdoid" pancreatic endocrine tumours were attributable to the accumulation of whorls of intermediate keratin filaments in a characteristic paranuclear or juxtanuclear location. These inclusions are analogous to keratin positive structures or "fibrous bodies" that are encountered in another endocrine gland, the pituitary. ${ }^{12}$ This should not be confused with Crooke's hyaline, which are aggregates of intermediate filaments that are dispersed throughout the cytoplasm and are not found as a characteristic paranuclear whorl or ball. The spectrum of morphological changes induced by fibrous bodies within the cytoplasm of endocrine neoplasms ranges from normal by light microscopy, to hyaline weakly eosinophilic inclusions, to deeply acidophilic/eosinophilic inclusions occupying most of the cytoplasm and displacing the nucleus eccentrically (the typical "rhabdoid" phenotype). Intracytoplasmic inclusions composed of aggregates of juxtanuclear cytokeratin immunoreactive intermediate filaments have been identified in several tumours of endocrine and related cells. Although the pathogenetic trigger in each different tumour may vary and remain conjectural at this juncture, juxtanuclear aggregates of intermediate cytokeratin filaments appear to be the common thread in most instances accounting for a similar morphological appearance in a variety of different tumours.

The purpose of our report is to highlight an example of a pancreatic endocrine tumour with an unusual morphological appearance, emphasise the spectrum of changes that can be obtained with cytoplasmic, juxtanuclear intermediate filament aggregation, and also to suggest that the term "rhabdoid" is not an apt description and should be replaced by the descriptive phrase "cytokeratin aggresomes" for the moment, until the exact clinical and biological relevance of this morphological appearance is better understood.

\section{CASE REPORT}

A 50 year old woman presented to the emergency department with acute onset of abdominal pain, nausea, and vomiting. Although the clinical impression was of viral gastroenteritis, the patient was admitted, given fluids, and 48 hours later her symptoms resolved. An ultrasound and computed tomography examination of the abdomen was ordered. Both confirmed a well circumscribed, lobulated, $4.4 \times 4.0 \times 3.6 \mathrm{~cm}$ mass in the region of the head of the pancreas, which appeared to be hypervascular with areas of microcalcification. No spread to the liver or lymph nodes was

Abbreviations: H\&E, haematoxylin and eosin; PET, pancreatic endocrine tumour 


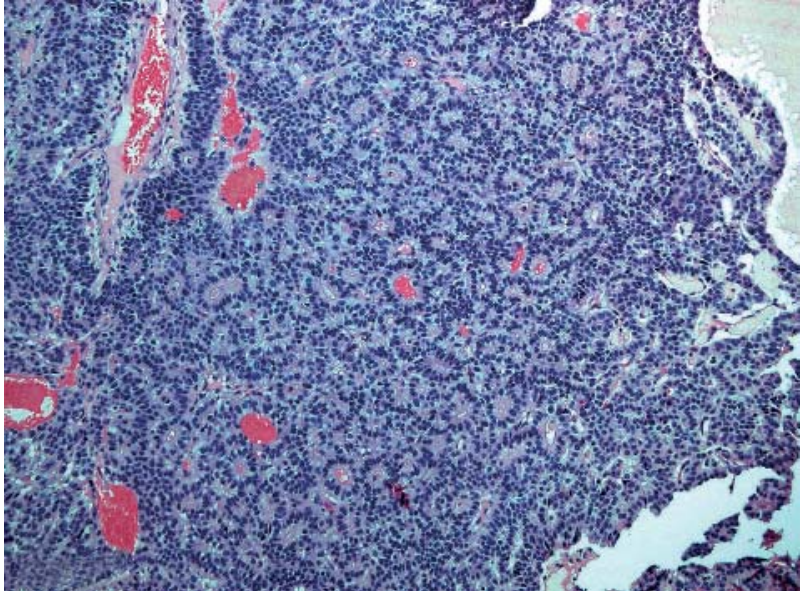

Figure 1 The tumour had a dominant tubulopapillary appearance with only focal solid and trabecular foci. Haematoxylin and eosin stain; original magnification, $\times 100$.

noted. In view of the clinical impression of carcinoma of the pancreas a Whipple's procedure was undertaken.

Postoperatively, the patient developed abdominal sepsis that responded to antibiotics. The rest of her postoperative course was uneventful and she was discharged.

\section{MATERIALS AND METHODS}

The surgical pathology specimen consisted of the distal portion of the stomach, duodenum, and jejunum, the head of the pancreas, and the cystic duct. The specimen was dissected as per protocol and sections of routinely processed formalin fixed, paraffin wax embedded tissue were stained with H\&E. A periodic acid Schiff preparation, with and without diastase digestion, and Southgate's mucicarmine stain were also done. In addition, immunohistochemistry was performed for the following antibodies/antigens: chromogranin (Biomeda, Foster City, California, USA), 1/400 dilution; synaptophysin (Dako, Ontario, Canada), 1/10 dilution; gastrin (Zymed, San Francisco, California, USA), 1/50 dilution; insulin (Dako), 1/4 dilution; glucagon (Novocastra, Newcastle upon Tyne, UK), 1/100 dilution; somatostatin (Zymed), 1/100 dilution; vasoactive intestinal peptide (Novocastra), 1/800 dilution; polypeptide P (Dako), 1/2000 dilution; CAM 5.2 (Becton Dickinson, San Jose, California, USA), 1/50 dilution; AEl/AE3 (Dako), 1/500 dilution; vimentin (American Research Products Inc, Belmont, Massachusetts, USA), 1/1200 dilution; Ki-67 (Immunotech, Westbrook, Maine, USA), 1/20 dilution.

Tissue was removed from a representative paraffin wax block and processed for electron microscopic examination.

\section{GROSS PATHOLOGY}

The stomach and duodenum were grossly unremarkable. The head of the pancreas measured $5.0 \times 5.5 \times 3.0 \mathrm{~cm}$, and the superior aspect contained a well circumscribed tumour that measured $4.5 \times 4.0 \times 3.0 \mathrm{~cm}$. On section, the tumour was firm, lobulated, glistening, and brown/tan to white in colour. The common bile duct measured $4.5 \mathrm{~cm}$ in length and $1.0 \mathrm{~cm}$ in circumference and was grossly unremarkable. The tumour did not compress or extend into the common bile duct. Fourteen lymph nodes were processed for histological evaluation.

\section{MICROSCOPY}

The tumour was arranged predominantly in a microglandular or tubulo/microacinar pattern, with focal trabecular and solid

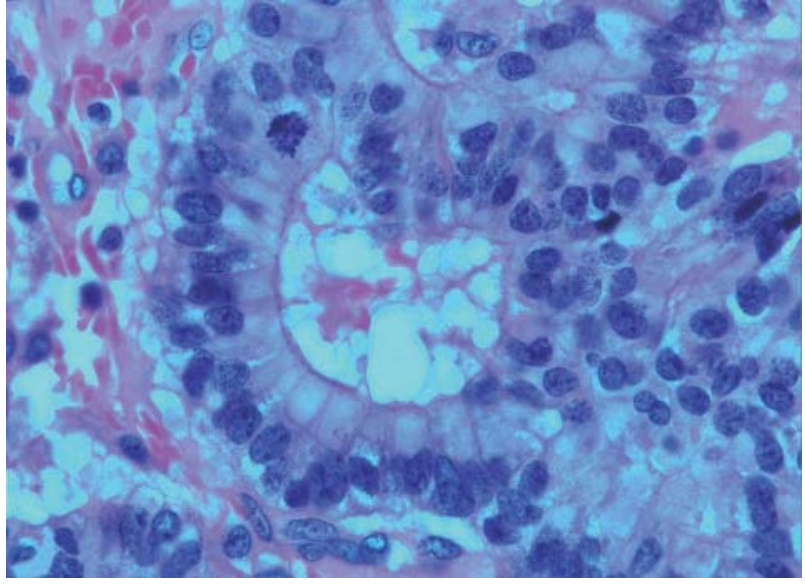

Figure 2 The inclusions were present in the luminal aspect of the cytoplasm, causing the cells to appear elongated and tall. Haematoxylin and eosin stain; original magnification, $\times 400$.

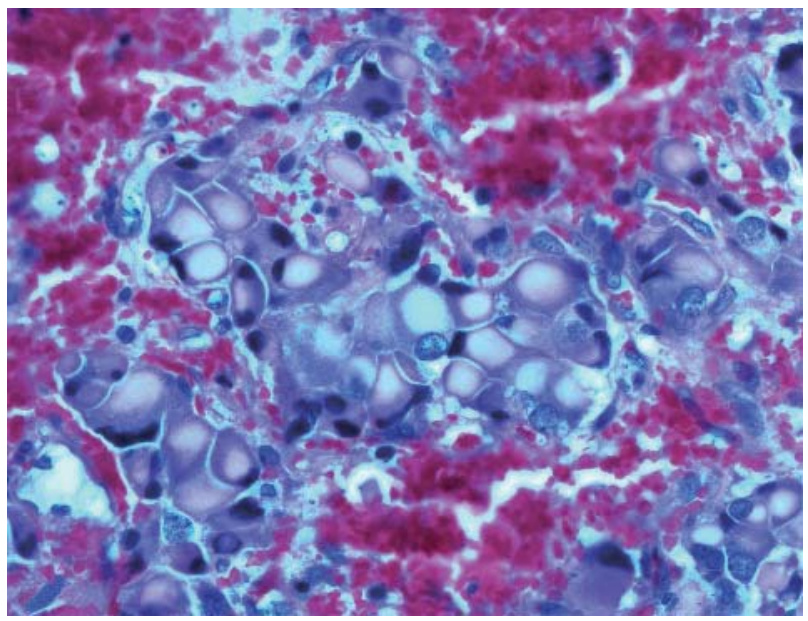

Figure 3 Some of the intracytoplasmic inclusions were very pale and weakly eosinophilic. In these cells the inclusions imparted a signet ring appearance to the cells. Haematoxylin and eosin stain; original magnification, $\times 400$

areas (fig 1). Within the microglandular structures the nuclei were basally oriented, with the apical portions of the cells containing abundant lightly eosinophilic, waxy hyaline material, organised into globules or inclusions within the cytoplasm. The hyaline inclusions occupied the luminal aspect of the microacinar structures and imparted an elongated appearance to the cells (fig 2). In some cells, the inclusions were sufficiently large to displace the nucleus eccentrically, resulting in a plasmacytoid or signet ring-like appearance (fig 3). In other cells, the inclusions assumed the more typical "rhabdoid" morphology, with deeply eosinophilic cytoplasmic inclusions and a peripherally displaced nucleus (fig 4). The inclusions were relatively uniform in size and distribution within the tumour cells. Cells arranged in trabeculae and solid sheets also contained inclusions. Extracellular hyaline was not seen. Those cells not containing inclusions had the typical finely granular, eosinophilic cytoplasm of classic endocrine cells. The nuclear chromatin had a "salt and pepper" distribution and several cells had small but distinct nucleoli. There was mild nuclear pleomorphism and the mitotic count ranged from four to nine/10 high power fields. Foci of necrosis with cholesterol clefts and 
foamy macrophages were present. The tumour infiltrated into fibrous tissue that was extremely hyalinised and calcified in areas, and into peripancreatic fibroadipose tissue. Both perineural and lymphatic permeation were noted. The hyaline inclusions were negative with the periodic acid Schiff, with and without diastase digestion, and mucin stains. All lymph nodes showed reactive change but no metastatic tumour. All resection margins were free of tumour.

The tumour cells were strongly positive for epithelial markers (CAM 5.2 and AEl/3); these keratin stains highlighted a conspicuous dot-like or globular staining pattern of the hyaline inclusions (fig 5). Vimentin also highlighted the cytoplasmic globules. In addition, synaptophysin was diffusely positive, whereas chromogranin A was only focally positive. The Ki-67 labelling index was approximately 6\%. All other markers were negative. The tumour was diagnosed as a low grade, non-functional, malignant endocrine tumour, according to the World Health Organisation classification.

Electron microscopy revealed the presence of aggregates of juxtanuclear intermediate filaments with entrapped neurosecretory granules (fig 6).

\section{DISCUSSION}

A wide array of cytoskeletal abnormalities is encountered at different sites and under different pathological conditions. Benign and malignant conditions of the liver, ovarian tumours, renal cell carcinomas, renal oncocytomas, soft tissue sarcomas, and central nervous system tumours have all been noted to contain hyaline bodies of some type. ${ }^{14}$

Thus far, only two reports of so called "rhabdoid" pancreatic endocrine tumours have appeared in the literature. ${ }^{56}$ In an analysis of pancreatic endocrine tumours in our records, cells of this morphology have been noted focally in occasional cases. However, the case presented in this report is the only one encountered thus far composed of a large population of "rhabdoid" cells.

The prototypic inclusion body in endocrine lesions is the fibrous body seen in some pituitary adenomas, especially the sparsely granulated somatotroph adenoma. ${ }^{15}$ The seminal observation of these juxtanuclear intermediate filaments was by Racadot and colleagues in $1964 .{ }^{16}$ The cells of the pituitary adenoma that they described were characterised by round hyaline masses often indenting or causing a concavity of the nucleus. ${ }^{16}$ Electron microscopic examination showed that these bodies consist of aggregates of intermediate filaments

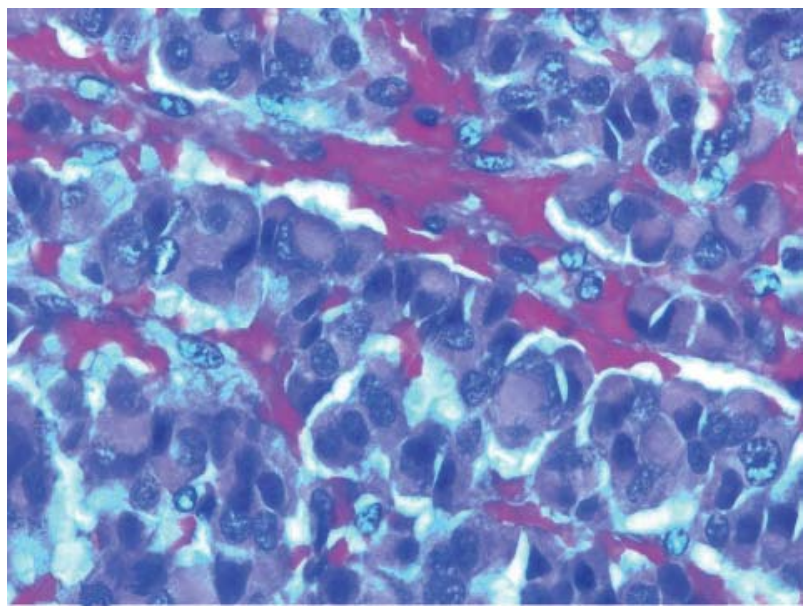

Figure 4 Cells exhibiting a more "rhabdoid" morphology with deeply eosinophilic cytoplasmic inclusions and a globular morphology. The nucleus is displaced peripherally. Haematoxylin and eosin stain; original magnification, $\times 400$.

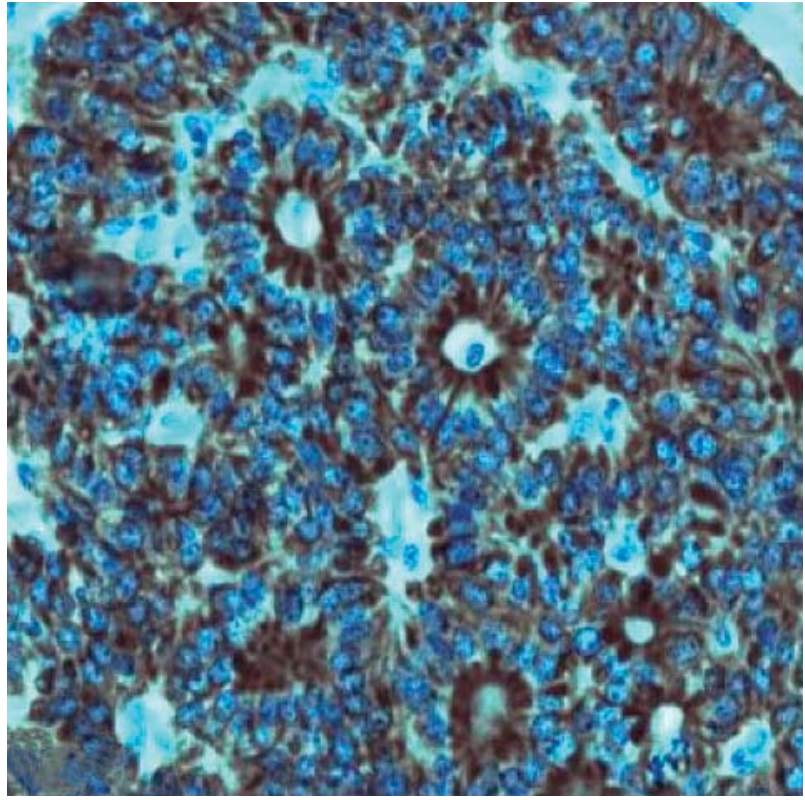

Figure 5 CAM 5.2 immunohistochemistry highlights the paranuclear globules of intermediate filaments. CAM 5.2 antibody; original magnification, $\times 200$.

in which occasional neurosecretory granules and mitochondria were also present. ${ }^{16}$ Cardell and Knighton were the first to use the term "fibrous bodies", which has become the most common and frequently used term to describe this phenomenon in the pituitary. ${ }^{17}$

Conditions in which cytoplasmic juxtanuclear aggregates of intermediate filaments are present are diverse and the pathogenesis of these structures is not known. It has been suggested that they are caused by cell damage and apoptosis. ${ }^{14}$ However, not all cells with intracytoplasmic inclusions exhibit evidence of damage. Indeed, in pituitary tumours, there is no correlation between the formation of

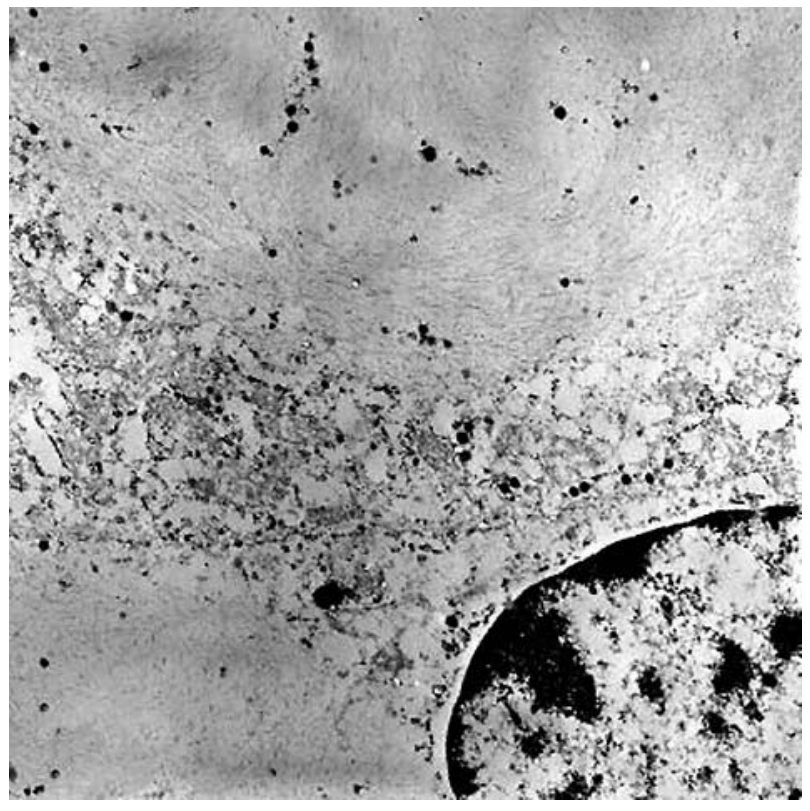

Figure 6 Electron microscopic illustration of a cell containing juxtanuclear whorls or bundles of intermediate filaments with occasional embedded neurosecretory granules. Original magnification, $\times 12000$. 
fibrous bodies and evidence of cell damage. Among pituitary growth hormone producing adenomas, the densely granulated tumours lack fibrous bodies; these lesions tend to occur in younger patients, often express prolactin and the $\alpha$ subunit of glycoprotein hormones, harbour Gs $\alpha$ mutations, ${ }^{18}$ and respond well to somatostatin analogue treatment. ${ }^{19}$ In contrast, sparsely granulated somatotroph adenomas are diagnosed on the basis of the fibrous body formation ${ }^{20}$; these lesions tend to occur in older patients, are rarely plurihormonal, do not harbour Gs $\alpha$ mutations, and do not respond well to somatostatin analogues. ${ }^{19}$

The appearance of these filamentous structures in $\mathrm{H} \& \mathrm{E}$ stained sections is variable; some tumours may not have detectable inclusions within the cytoplasm. They may be seen on toluidine blue stained, plastic embedded, semi-thin sections as a well defined, pale, globoid cytoplasmic inclusion. In some cases, they represent an ultrastructural observation only. However, keratin immunohistochemistry highlights the presence of juxtanuclear globular structures. At the ultrastructural level, intracytoplasmic fibrillary inclusions or whorls have been noted in endocrine tumours of the bronchus and rectum, paragangliomas of the cauda equina, ${ }^{13}$ and in an endocrine tumour of the pancreas. ${ }^{21}$ Paranuclear or juxtanuclear intermediate filaments are also characteristic of Merkel cell neuroendocrine carcinomas.

\section{"We advocate the use of the descriptive term cytokeratin aggresomes for tumours containing juxtanuclear aggre- gates of keratin filaments, because this is more reflective of the structural abnormality causing the characteristic morphological appearance"}

The term "rhabdoid" has been used to describe tumours composed of cells with globular cytoplasmic inclusions that are intensely eosinophilic/acidophilic. ${ }^{22}$ It is obviously reflective of the striking similarity and resemblance to rhabdomyoblasts. Thus, a recently described case similar to the one we present was identified as a "rhabdoid" pancreatic neuroendocrine carcinoma. ${ }^{5}$ The nomenclature describes a "rhabdomyoblast-like" appearance but implies that the cells are not true rhabdomyoblasts. The term has been further exploited to describe "rhabdoid-like" inclusions-pale, lightly eosinophilic cytoplasmic inclusions within a deeply eosinophilic cytoplasm that are keratin negative and are composed of both thick and thin filaments, not intermediate filaments. ${ }^{23}$ In contrast, the "signet ring cell" pancreatic tumour described by Stokes and colleagues ${ }^{11}$ was composed of cells with inclusion bodies consisting of multilayered, concentric, osmiophilic lamellae or myelin figures, ${ }^{11}$ ascribed to an abnormal accumulation of degenerating organelles. These features are different from the hyaline or plasmacytoid cells seen in myoepitheliomas and pleomorphic adenomas of salivary glands, ${ }^{24} 25$ in which the cells have a diffusely hyaline, eosinophilic cytoplasm with an eccentrically located nucleus, but do not have hyaline globular, inclusion-like bodies. The cell morphology that is highlighted in this case is also different from the commonly encountered "plasmacytoid" cells seen with air dried Diff-Quik stained fine needle aspiration specimens of endocrine tumours. ${ }^{26}$ Once again, cytoplasmic inclusions are not present.

The widespread use of "rhabdoid" as a purely descriptive term is somewhat entrenched in the literature, with an ever expanding list of tumours ("composite extrarenal rhabdoid tumours") exhibiting this characteristic cell type. The resemblance to rhabdomyoblasts is superficial and based on morphological mimicry, and "rhabdoid" does not reflect histogenesis or cell structure. In fact, it is merely a convenient term. In this context, it loses relevance and may lead to the
Take home messages

- We describe a pancreatic endocrine tumour with an unusual morphological appearance

- This case highlights the spectrum of changes that can be seen with cytoplasmic, juxtanuclear intermediate filament aggregation and that the application of the term "rhabdoid" as an all embracing term is inappropriate

- Use of the term "cytokeratin aggresomes" has the advantage of alluding to the pathological feature, rather than implying histogenesis, and unifies the identical process that occurs across several tumours

erroneous interpretation of histogenesis in tumours harbouring cells with a similar morphology. We advocate the use of the descriptive term "cytokeratin aggresomes" for tumours containing juxtanuclear aggregates of keratin filaments, because this is more reflective of the structural abnormality causing the characteristic morphological appearance. ${ }^{27}$ This term was first used by Johnston and colleagues to describe the redistribution of the intermediate filament protein vimentin to form a cage surrounding a pericentriolar core of aggregated, ubiquitinated protein. ${ }^{27}$ We feel that this phenomenon is especially true in the case of tumours with a neuroendocrine histogenesis, and because the same process occurs in the pituitary, the use of "cytokeratin aggresomes" is more inclusive and unifying. The exact incidence and relevance of cells bearing this characteristic morphology in pancreatic endocrine tumours is not clear at this juncture.

The use of the term "rhabdoid" in pancreatic endocrine tumours especially is not a particularly apt appellation, and is not necessarily reflective of histogenesis. The light microscopy of routinely stained H\&E sections of some PETs may be normal, but there may be immunohistochemical and/or ultrastructural evidence of intracytoplasmic whorls of intermediate filaments. In other cases, cytoplasmic inclusions may be detected in individual cells or they may be highly conspicuous on routine sections examined by light microscopy. It is important to point out that a range of morphological appearances can be encountered as a result of cytoplasmic aggregates of intermediate filaments; some cells resembling signet ring cells and others "rhabdoid" cells. The case described here illustrates that intracytoplasmic juxtanuclear intermediate filaments can cause a range of cytomorphological appearances and that the application of the term "rhabdoid" as an all embracing term is inappropriate. The use of the term "fibrous bodies" (as seen and used in the pituitary) may also be inappropriate. "Cytokeratin aggresomes" does have the advantage of alluding to the pathological feature, rather than implying histogenesis, and does unify the identical process that occurs across several tumours.

\section{Authors' affiliations}

R Chetty, S L Asa, Department of Pathology, University Health Network/ Toronto Medical Laboratories, University of Toronto, Toronto, ON M5G $2 \mathrm{M} 9$, Canada

Correspondence to: Professor R Chetty, University Health Network, Princess Margaret Hospital, 610 University Avenue, Fourth Floor, Suite 302, Room 312, Toronto, ON M5G 2M9, Canada; runjan.chetty@uhn. on.ca

Accepted for publication 9 May 2004 


\section{REFERENCES}

1 Gotchall J, Traweek ST, Stenzel P. Benign oncocytic endocrine tumour of the pancreas in a patient with polyarteritis nodosa. Hum Pathol 1987; 18:967-9.

2 Pacchioni D, Papotti M, Macri L, et al. Pancreatic oncocytic endocrine tumours. Cytologic features of two cases. Acta Cytol 1996;40:742-6.

3 Lubensky IA, Pack S, Ault D, et al. Multiple neuroendocrine tumours of the pancreas in von Hippel-Lindau disease. Am J Pathol 1998;153:223-31.

4 Hoang MP, Hruban R, Albores-Saavedra J. Clear cell endocrine pancreatic tumour mimicking renal cell carcinoma: a distinctive neoplasm of Von HippelLindau disease. Am J Surg Pathol 2001;25:602-9.

5 Perez-Montiel MD, Frankel WL, Suster S. Neuroendocrine carcinomas of the pancreas with "rhabdoid" features. Am J Surg Pathol 2003;27:642-9.

6 Shia J, Erlandson RA, Klimstra DS. Whorls of intermediate filaments with entrapped neurosecretory granules correspond to the "rhabdoid" inclusions seen in pancreatic endocrine neoplasms. Am J Surg Pathol 2004; $28: 271-3$

7 Lack EE. Pathology of the pancreas, gallbladder, extrahepatic biliary tract and ampullary region. Pancreatic endocrine neoplasms, 1st ed. New York: Oxford University Press, 2003:323-73.

8 Ordonez NG, Balsaver AM, Mackay B. Mucinous islet cell (amphicrine) carcinoma of the pancreas associated with watery diarrhea and hypokalemia syndrome. Hum Pathol 1988;19:1458-61.

9 Tomita T, Bhatia P, Gourley W. Mucin producing islet cell adenoma. Hum Pathol 1981;12:850-3.

10 Terada R, Ito S, Akama F, Kashima K, et al. Small nonfunctioning islet cell tumour in the body of the pancreas: report of a case. Surg Today 2004:34:177-80.

11 Stokes MB, Kumar A, Symmans WF, et al. Pancreatic endocrine tumour with signet ring cell features: a case report with novel ultrastructural observations. Ultrastruct Pathol 1998;22:147-52.

12 Cheung CC, Asa SL. Pathology of the pituitary gland. In: LiVolsi VA, Asa SL, eds. Endocrine pathology. Philadelphia: Churchill Livingstone, 2002:31-59.

13 Neumann PE, Goldman JE, Horoupian DS, et al. Fibrous bodies in growth hormone-secreting adenomas contain cytokeratin filaments. Arch Pathol Lab Med 1985; 109:505-8.
14 Papadimitriou JC, Drachenberg CB, Brenner DS, et al. "Thanatosomes": a unifying morphogenetic concept for tumour hyaline globules related to apoptosis. Hum Pathol 2000;31:1455-65.

15 Asa SL. Tumors of the pituitary gland. In: Rosai J, ed. Atlas of tumour pathology, Fascicle 22, Third Series. Washington DC: Armed Forces Institute of Pathology, 1998:54-71.

16 Racadot J, Olivier L, Porcile E, et al. Adenome hypophysaire de type "mixte" avec symptomologie acromegalique: II. Etude au microscope Optique et au microscope electronique. Ann Endocrinol 1964;25:503-7.

17 Cardell RR, Knighton RS. The cytology of a human pituitary tumour: an electron microscopic study. Trans Am Microsc Soc 1966;85:58-78.

18 Spada A, Arosio M, Bochicchio D, et al. Clinical, biochemical and morphological correlates in patients bearing growth hormone-secreting pituitary tumours with or without constitutively active adenylyl cyclase. J Clin Endocrinol Metab 1990;71:1421-6.

19 Ezzat S, Kontogeorgos G, Redelmeier DA, et al. In vivo responsiveness of morphological variants of growth hormone-producing pituitary adenomas to octreotide. Eur J Endocrinol 1995; 133:686-90.

20 Sano T, Ohshima, T, Yamada S. Expression of glycoprotein hormones and intracytoplasmic distribution of cytokeratin in growth hormone-producing pituitary adenomas. Pathol Res Pract 1991;187:530-3.

21 Ordonez NG. Insulinoma with fibrillar inclusions and acinar cell elements Ultrastruct Pathol 2001;25:485-95

22 Nappi O, Ferrara G, Wick MR. Neoplasms composed of eosinophilic polygonal cells: an overview with consideration of different cytomorphologic patterns. Semin Diagn Pathol 1999;16:82-90.

23 Perez-Ordonez B, Kandel RA, Bell R, et al. Rhabdomyosarcoma with rhabdoid-like features. Pathol Res Pract 1998;194:357-61.

24 Buchner A, David R, Hansen LS. "Hyaline" cells in pleomorphic adenoma of salivary gland origin. Oral Surg Oral Med Oral Pathol 1981;52:506-12.

25 Lomax-Smith JD, Azzopardi JG. The hyaline cell: a distinctive feature of "mixed" salivary tumours. Histopathology 1978;2:77-92.

26 Crapanzano JP. Cytology of low-grade endocrine neoplasms involving liver: a series of 24 specimens, including 4 with hepatoid or glandular features. Diagn Cytopathol 2004;30:31-8.

27 Johnston JA, Ward CL, Kopito RR. Aggresomes: a cellular response to misfolded proteins. J Cell Biol 1998;143:1883-98. 